\title{
Oxyarietites boletzkyi n. gen., n. sp., nouveau genre et nouvelle espèce d'ammonite dans le Sinémurien inférieur de Bourgogne (France): un rare précurseur des morphologies oxycônes pour le Jurassique
}

\author{
Jean-Louis DOMMERGUES \\ UFR Sciences de la Vie, de la Terre et de l'Environnement, \\ Université de Bourgogne, CNRS/uB, UMR 6682, Biogéosciences Dijon, \\ 6 Boulevard Gabriel, F-21000 Dijon (France) \\ jean-louis.dommergues@u-bourgogne.fr \\ Stijn GOOLAERTS \\ Geo-Instituut, \\ Celestijnenlaan $200 \mathrm{E}$, \\ B-3001 Heverlee (Belgique) \\ stijn.goolaerts@ees.kuleuven.be
}

\footnotetext{
MOTS CLÉS

Ammonites, Jurassique inférieur, stratigraphie, morphologie, adaptation, diversité, disparité, genre nouveau, espèce nouvelle.
}

Dommergues J.-L. \& Goolaerts S. 2012. - Oxyarietites boletzkyi n. gen., n. sp., nouveau genre et nouvelle espèce d'ammonite dans le Sinémurien inférieur de Bourgogne (France): un rare précurseur des morphologies oxycônes pour le Jurassique. Geodiversitas 34 (3): 517-529. http://dx.doi.org/10.5252/g2012n3a4

\section{RÉSUMÉ}

L'une des toutes premières ammonites à coquille presque oxycône observée dans les séries fossiliferes après la crise faunique de la limite Trias/Jurassique est décrite. Elle provient du Sinémurien inférieur (chronozone à Semicostatum ou à Turneri) de Bourgogne (Mavilly-Mandelot, Côte-d'Or, France). Cette nouvelle forme, Oxyarietites boletzkyi n. gen., n. sp., possède une coquille involute, comprimée et carénée dont le type clairement suboxycône est nouveau pour le Sinémurien inférieur. En raison de son aire ventrale carénée, ce taxon se rattache probablement à la super-famille des Arietitoidea Hyatt, 1875 sensu Guex (1995) mais son attribution familiale est incertaine et son origine évolutive reste énigmatique. Outre son intérêt taxonomique, cette découverte est importante car elle pose le problème de la valeur adaptative des caractères liés à la géométrie des coquilles d'ammonites. Il est actuellement admis que les coquilles involutes, comprimées et carénées de type suboxycône et oxycône favorisent significativement l'hydrodynamisme et donc la mobilité des espèces qui les possèdent. Il est surprenant que l'acquisition de ce probable avantage adaptatif n'ait pas favorisé l'implantation au sein des peuplements du nouveau taxon, qui reste une forme rare. Dans tous les cas, la découverte d'O. boletzkyi n. gen., n. sp. rajeunit d'environ 2 millions d'années la mise en place des morphologies oxycônes au cours de la phase de reconstitution de la biodiversité post-crise Trias/Jurassique. 


\author{
KEY WORDS \\ Ammonites, \\ Early Jurassic, \\ stratigraphy, \\ morphology, \\ adaptation, \\ diversity, \\ disparity, \\ new genus, \\ new species.
}

\begin{abstract}
Oxyarietites boletzkyi $n$. gen., n. sp., a new genus and species of ammonite for the Lower Sinemurian of Burgundy (France): a rare forerunner of the oxycone morphologies for the Jurassic.

One of the very first quasi-oxycone ammonites following the Triassic/Jurassic boundary crisis is described. It was collected from the fossiliferous Lower Sinemurian (Semicostatum or Turneri Chronozone) strata of Burgundy (Mavilly-Mandelot, Côte-d'Or, France). The new taxon, Oxyarietites boletzkyi n. gen., n. sp., possesses an involute, compressed and keeled shell of suboxycone morphology, a shell type previously unknown for the Lower Sinemurian. The discovery makes younger by about $2 \mathrm{Ma}$ the emergence of keeled (sub)oxycone shells following the Triassic/Jurassic boundary crisis. Its obviously keeled ventral area allows a probable assignation to the Arietitoidea Hyatt, 1875 sensu Guex (1995) superfamily, but family level assignation and its evolutionary origin remain obscure. Although, it is generally accepted that involute, compressed and keeled suboxycone and oxycone ammonite shells possess the best hydrodynamical abilities and mobility, the acquisition of this probable adaptive advantage in $O$. boletzkyi n. gen., n. sp. does not go together with abundancy in the fossil record.
\end{abstract}

\section{INTRODUCTION}

Les ammonites, apparues au Dévonien inférieur, ont connu, tant en termes de diversité que de disparité, une histoire évolutive spectaculaire et mouvementée. Le groupe a risqué l'extinction à la fin du Dévonien, du Permien et du Trias avant de disparaître définitivement à la fin du Crétacé. On peut donc grossièrement considérer que l'ensemble des ammonites comprend quatre ensembles distincts séparés par trois goulets d'étranglement majeurs (bottlenecks). Après chacun de ces évènements, la diversité et la disparité des ammonites se sont reconstituées en général assez rapidement à partir de quelques rares espèces voire d'une seule espèce. Dans le cas de la quasi-extinction de la limite Trias/ Jurassique $(\mathrm{Tr} / \mathrm{J})$, seuls quelques Phylloceratina Arkell, 1850 apparentés au genre Rhacophyllites Zittel, 1884 (Discophyllitidae Spath, 1927) semblent avoir traversé la limite $\operatorname{Tr} / \mathrm{J}$ (Tozer 1981; Guex 1982, 1987; Rakus 1993; Guex et al. 1998; Hillebrandt \& Krystyn 2009). Les modalités de la reconstitution post-crise $\mathrm{Tr} / \mathrm{J}$ de la diversité (richesse taxonomique) et de la disparité (formes et/ou tailles des coquilles) ont fait l'objet de plu- sieurs études récentes (Dommergues et al. 1996, 2001, 2002; Simon et al. 2010). Les ammonites étant un groupe d'importance stratigraphique majeur, le patron du redéploiement des faunes d'ammonites, au cours des trois premiers étages du Jurassique, semble maintenant assez bien établi. C'est notamment le cas en Europe du nord-ouest où la découverte d'un nouveau taxon est depuis longtemps devenue un évènement rare. De plus, la description d'une espèce nouvelle aboutit généralement à un simple enrichissement de la diversité taxonomique, sans conséquence notable sur les patrons de déploiement de la disparité. Parmi ceux-ci, l'apparition des coquilles oxycônes, nettement plus tardive que celles de tous les autres types morphologiques, est un fait remarquable. Il faut attendre environ sept millions d'années et la partie moyenne du Sinémurien supérieur (chronozone à Oxynotum) pour observer l'apparition dans le registre fossile des toutes premières formes oxycônes s.s. (e.g., Oxynoticeras Hyatt, 1875 ; Dommergues et al. 1996: figs 12, 13). La découverte d'une ammonite dont la coquille est presque oxycône, Oxyarietites boletzkyi n. gen., n. sp., au cours du Sinémurien inférieur (chronozone à Semicostatum) 
de Bourgogne (France) conduit donc à envisager une mise en place plus précoce d'environ deux millions d'années pour ce type morphologique. Le présent travail est dédié à la description de ce nouveau taxon, morphologiquement précurseur, et à l'analyse des implications taxonomiques, stratigraphiques et évolutives de sa découverte.

\section{CADRES GÉOGRAPHIQUE ET GÉOLOGIQUE}

Les ammonites étudiées dans le présent travail, et notamment le nouveau taxon Oxyarietites boletzkyi n. gen., n. sp., proviennent d'un affleurement correspondant à une tranchée de l'ancienne ligne ferroviaire dite du "Tacot de Saulieu» qui menait de Beaune à Semur-en-Auxois via Saulieu (Côte d'Or). La coupe est située sur la commune de Mavilly-Mandelot (Côte d'Or) à environ $300 \mathrm{~m}$ à l'ouest du hameau de Mandelot et un peu au-dessus de la route départementale D32d (044”'21'E, 4703'32”N) (Fig. 1). Cette coupe expose, sur environ trois mètres d'épaisseur, des assises du Sinémurien et du Pliensbachien inférieur. Certains niveaux sont très fossilifères. La coupe de Mandelot a fait l'objet de récoltes intensives et systématiques (ammonites, bélemnites, bivalves, brachiopodes) effectuées avec la précision du banc. Les résultats concernant les faunes d'ammonites ont fait l'objet de deux publications. La première est essentiellement dédiée au faunes du Sinémurien supérieur et du Pliensbachien inférieur (Dommergues 1993), la seconde à celles du Sinémurien inférieur (Corna \& Dommergues 1995).

Les faunes d'ammonites du Sinémurien de Mandelot sont localement abondantes et diversifiées. Elles comprennent une trentaine d'espèces correspondant aux chronozones à Bucklandi (partie terminale), Semicostatum, Turneri et Obtusum (partie basale) (Fig. 2). Une lacune importante correspondant aux chronozones à Obtsusum (partie basale exceptée), Oxynotum, Raricostatum, Jamesoni et sans doute aussi Ibex (partie terminale exceptée), sépare la série sinémurienne des assises du Pliensbachien inférieur. Cette lacune

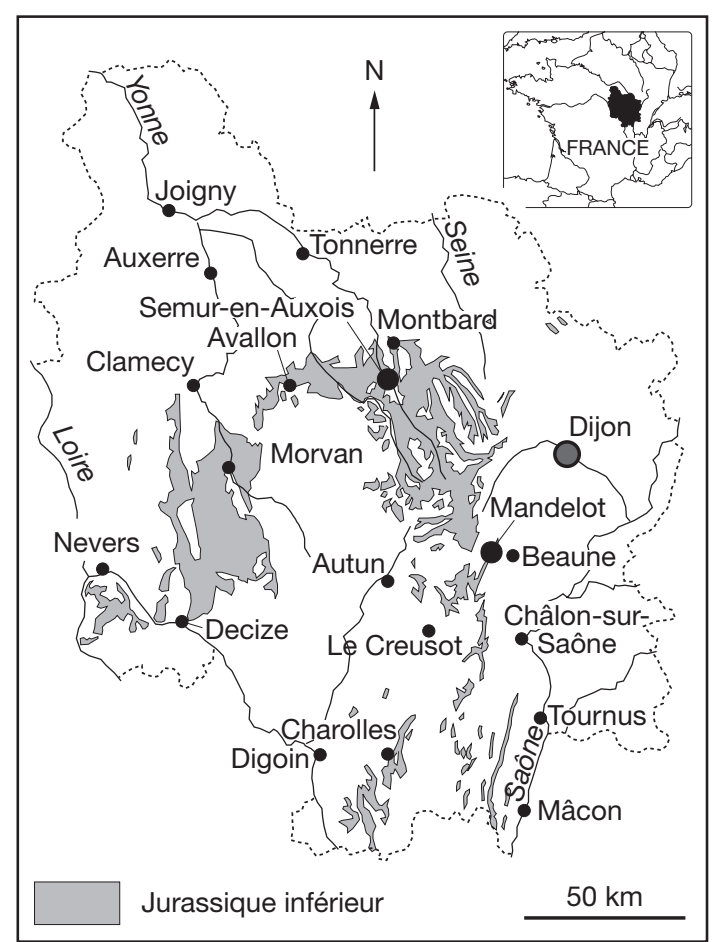

FIG. 1. - Cadre géographique et géologique schématique de la Bourgogne et localisation du gisement de Mandelot (Côte-d'Or) au SW de Dijon. La ville de Semur-en-Auxois (Côte-d'Or), choisie par d'Orbigny (1849: 157) comme localité de référence pour le Sinémurien, second étage du Jurassique, est également indiquée.

correspond à une nette discontinuité lithologique située entre les niveaux 98 sup. et 99 (Fig. 2). De part et d'autre de cette discontinuité, le contraste lithologique est important entre la série sinémurienne de type "Calcaires à gryphées" (Fig. 2; niveau 74-98 sup.) et les assises condensées du Pliensbachien inférieur qui se réduisent pour l'essentiel à un banc massif de calcaire clair à bélemnites et pseudo-oolites ferrugineuses (Fig. 2; niveau 99-101).

Au sein de la série relativement monotone des "Calcaires à gryphées" du Sinémurien, le niveau 96, et plus particulièrement sa partie moyenne, d'où provient Oxyarietites boletzkyi n. gen., n. sp., se distingue par l'abondance de coquilles gris clair, souvent phosphatées d'ammonites, de bivalves et de brachiopodes qui contrastent fortement avec la gangue sombre et finement 
bioclastique. Relativement gélif, le niveau 96 moyen affleure souvent en retrait par rapport aux niveaux qui l'encadrent. En termes bio- et chronostratigraphiques, le niveau 96 moyen est condensé. Les espèces qu'il contient peuvent être attribuées à la partie supérieure de la chronozone à Semicostatum (sous-chronozones à Scipionianum et/ou à Sauzeanum) et/ou à la base de la chronozone à Turneri.

Les "Calcaires à gryphées» du Sinémurien de Bourgogne sont souvent assez fossilifères et les affleurements, naturels ou artificiels, propices aux études paléontologiques et stratigraphiques, ne sont pas rares. Certains secteurs particulièrement favorables sont connus et étudiés depuis le dix-neuvième siècle. D’Orbigny (1849: 157) a même désigné la ville de Semur-en-Auxois (du latin Sene Muros ou "Vieilles murailles») située au nord de la Côte d'Or (Fig. 1) comme localité éponyme pour le Sinémurien (deuxième étage du Système Jurassique). Dans sa «Paléontologie française " d'Orbigny (1844) a également illustré des ammonites du Sinémurien de Bourgogne. Ces faunes ont été récemment révisées (Fischer 1994). Après les travaux de d'Orbigny (1844, 1849), les dépôts du Sinémurien aux environs de Semur ont fait l'objet des recherches de Collenot $(1873,1879)$ dont les importantes collections ont été en grande partie révisées par Guérin-Franiatte (1966, 1990). Plus récemment, l'étude des séries du Sinémurien de Bourgogne a été reprise par Mouterde (1953), Mouterde \& Tintant (1961, 1964, 1980), Corna \& Mouterde (1988), Dommergues (1993) et Corna \& Dommergues (1995) et de nouvelles collections d'ammonites, souvent récoltées avec la précision du banc, ont alors été réunies. On peut donc considérer que la connaissance du Sinémurien de Bourgogne est actuellement assez avancée et la découverte d'un nouveau taxon, morphologiquement très original pour son niveau stratigraphique, mérite d'être soulignée.

Plus généralement, et depuis le dix-neuvième siècle, le Sinémurien du nord-ouest de l'Europe a été intensivement exploré (e.g., France, Allemagne et Grande Bretagne) et la description d'un nouveau taxon provenant de ce domaine paléogéographique est devenue un évènement rare.

\section{SYSTÉMATIQUE}

Classe CEPHALOPODA Cuvier, 1798

Sous-classe AMMONOIDEA Zittel, 1884

Ordre PHYLLOCERATIDA Arkell, 1950

\section{REMARQUES}

L'ordre des Phylloceratida, pris ici au sens de Hoffmann (2010), est un taxon monophylétique qui inclus le sous-ordre des Phyllocertina Arkell, 1950 et l'ensemble des formes qui en dérivent. Ces dernières sont regroupées au sein du sous-ordre des Psiloceratina Houša, 1965 sensu Guex (1995). Au sens de Hoffmann (2010), l'ordre des Phylloceratida peut être compris comme un clade incluant deux sous-ordres, l'un paraphylétique correspond aux Phylloceratina et l'autre très probablement monophylétique correspondant aux Psiloceratina (= Ammonitina Zittel, 1884 sensu Hoffmann 2010) qui s'enracinent vers la limite Trias-Jurassique au sein des Phylloceratina.

Sous-ordre PSILOCERATINA Houša, 1965 Super-famille ARIETITOIDEA Hyatt, 1875 sensu Guex (1995)

Famille Incertae sedis

\section{REMARQUeS}

L'attribution d'Oxyarietites boletzkyi n. gen., n. sp. à la super-famille des Arietitoidea est probable mais reste hypothétique. En effet, la coquille presque oxycône de la nouvelle forme s'écarte considérablement des morphologies exprimées par les autres représentants de cette super-famille et aucune transition évolutive n'est actuellement connue.

\section{Genre Oxyarietites n. gen.}

ESPÈCE TYPE ET UNIQUE ESPÈCE. - Oxyarietites boletzkyi n. sp.

ETYMOLOGIE.- Le préfixe " oxy" évoque la morphologie presque oxycône de la coquille du nouveau taxon et la racine "arietites" indique sa probable affinité avec les Arietitoidea. 


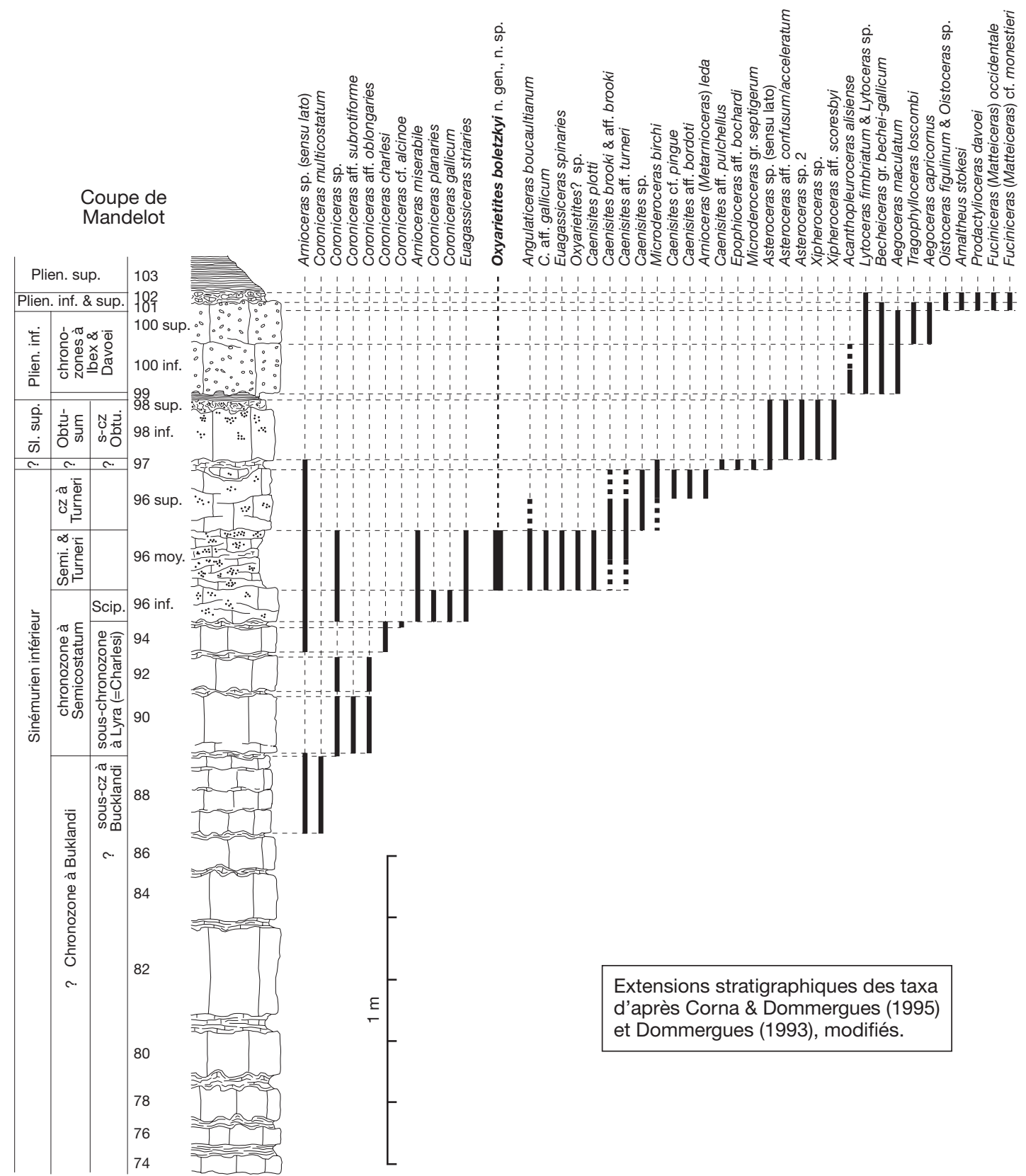

FIG. 2. - Coupe des assises du Sinémurien et du Pliensbachien affleurant le long de l'ancienne voie ferrée dites du «Tacot » à l'ouest du village de Mandelot (Côte-d'Or). Le profil lithologique d'érosion, la distribution des ammonites et l'interprétation chronostratigraphique sont une synthèse basée sur les travaux de Dommergues (1993) et Corna \& Dommergues (1995), ainsi que sur des observations et des récoltes récentes. Le nouveau taxon Oxyarietites boletzkyi n. gen., n. sp. (en gras) provient du niveau 96 moyen. Échelle chronostratigraphique d'après Corna et al. (1997) et Page (2003). 

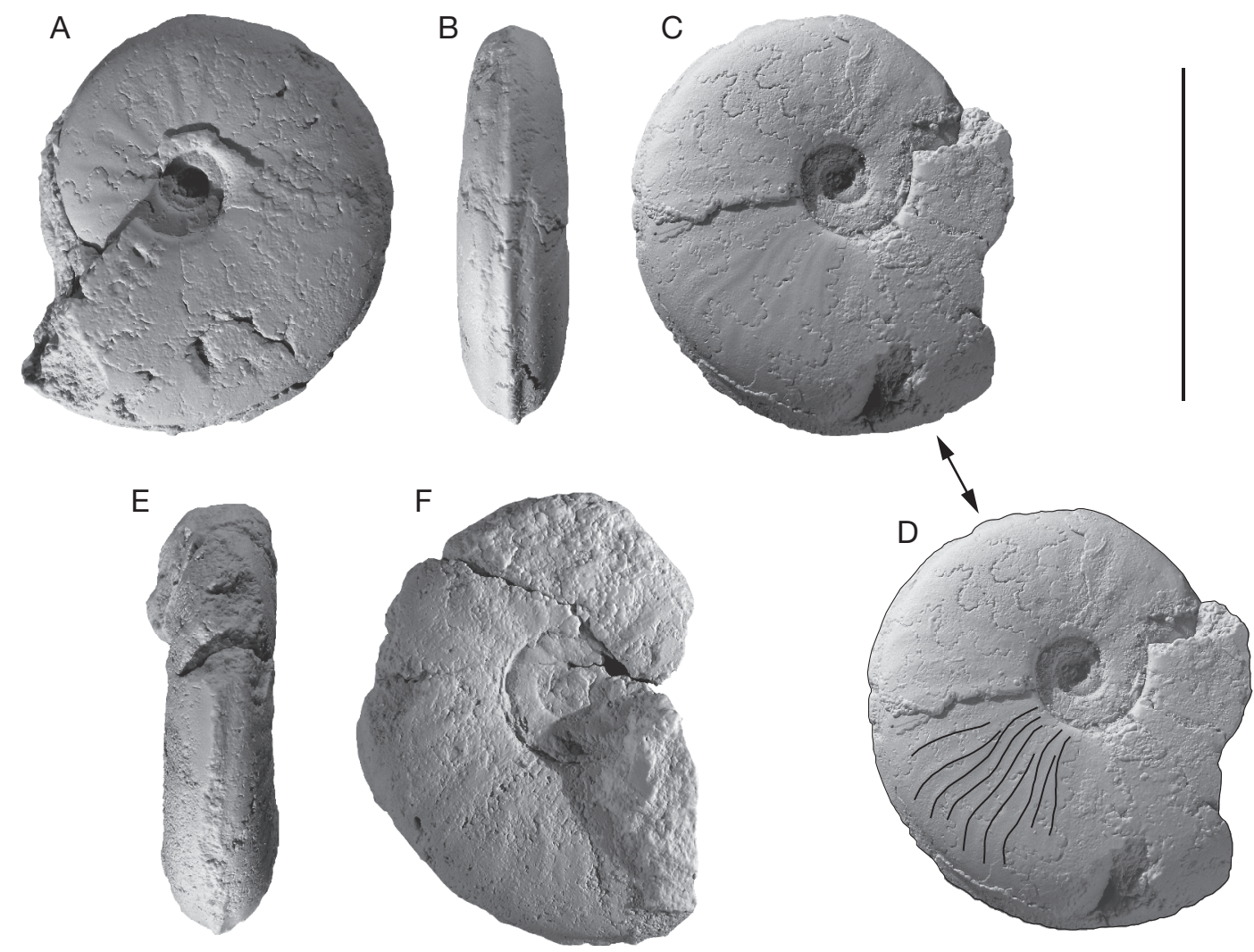

FIG. 3. - Ammonites à morphologie suboxycône ou subplatycône involute provenant du niveau 96 moyen de la coupe de Mandelot (Côte-d'Or France) (Sinémurien inférieur), chronozone à Semicostatum (sous-chronozone à Scipionianum ou à Sauzeanum) ou chronozone à Turneri. A-D, Oxyarietites boletzkyi n. gen., n. sp., holotype (UBGD 277446); D, interprétation graphique de la costulation. E, F, Oxyarietites? sp. (Man-96moy-001). Échelle: $2 \mathrm{~cm}$.

DiAGNOSE. - Coquille presque oxycône avec un ombilic de petit diamètre et peu profond. La section des tours est nettement comprimée. Le taux de recouvrement des tours (c. $35 \%)$ est important. Le rebord ombilical est bien marqué. Il correspond à une brusque courbure de la coquille qui sépare nettement la base des flancs, presque parallèles dans leur partie inférieure, du mur ombilical. Celui-ci, est peu développé mais quasiment perpendiculaire au plan d'enroulement de la coquille. Laire ventrale ogivale, presque anguleuse, porte une carène peu tranchante mais bien individualisée bordée par deux méplats obliques assez larges. La ligne de suture au tracé globalement rétroverse est faiblement découpée avec un lobe suspensif ample mais peu incisé et d'allure presque plate. L'ornementation latérale est très discrète, mais en lumière rasante, on peut observer une costulation délicate formée de côtes au tracé flexueux. Ces côtes tendent à se diviser vers le tiers inférieur des flancs.

\section{Oxyarietites boletzkyi n. sp. \\ (Figs 3A-D; 4A; 5A, B)}

HoLOTYPE. - UBGD 277446. Moule interne phosphaté de $24,5 \mathrm{~mm}$ de diamètre et sa contre-empreinte. Il s'agit d'un phragmocône finement préservé mais incomplet car entièrement cloisonné et sans approximation évidente des dernières cloisons visibles. L'holotype est le seul exemplaire connu. Il est conservé dans les collections de paléontologie de l'Université de Bourgogne (code UBGD).

Mesures. - Deux séries de mesures ont été prises sur le dernier tour de l'holotype. Elles sont données sur le Tableau 1.

ÉTYMOLOGIE. - En l'honneur de Sigurd von Boletzky pour son exceptionnelle connaissance du monde des céphalopodes. 
TABLEAU 1. - Oxyarietites boletzkyi n. gen., n. sp., mesure de I'holotype (UBGD 277446). Abréviations: D, diamètre; H, hauteur du tour; E, épaisseur du tour; R, rayon; O, ombilic. Les dimensions linéaires $(D, H, E, R$ et $O)$ sont exprimées en millimètres. À l'exception du rapport $E / H$, les indices morphologiques $(H / D, E / D$, $\mathrm{R} / \mathrm{D}$ et $\mathrm{O} / \mathrm{D}$ ) sont donnés en pourcentages du diamètre.

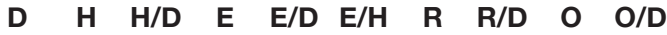

$\begin{array}{llllllllllllllll}26,4 & 11,8 & 45 \% & 7,1 & 27 \% & 0,60 & 15,0 & 57 \% & 5,3 & 20 \%\end{array}$ $\begin{array}{llllllllll}21,2 & 10,2 & 48 \% & 6,3 & 30 \% & 0,69 & 12,1 & 57 \% & 4,0 & 19 \%\end{array}$

TABLEAU 2. - Série de mesures prises sur le tour externe d'Oxyarietites? sp. (Man-96 moy-001). Abréviations: D, diamètre; $\mathbf{H}$, hauteur du tour; $\mathbf{E}$, épaisseur du tour; $\mathbf{R}$, rayon; $\mathbf{O}$, ombilic. Les dimensions linéaires ( $D, H, E, R$ et $O)$ sont exprimées en millimètres. À l'exception du rapport $\mathrm{E} / \mathrm{H}$, les indices morphologiques $(H / D, E / D, R / D$ et $O / D)$ sont donnés en pourcentages du diamètre.

\begin{tabular}{cccccccccc}
\hline D & H & H/D & E & E/D & E/H & R & R/D & O & O/D \\
\hline 23,4 & 9,4 & $40 \%$ & 6,3 & $27 \%$ & 0,67 & - & - & 7,4 & $31 \%$ \\
\hline
\end{tabular}

Localité et STRATE TyPes. - Mandelot, Côte-d'Or, France. Coupe du Tacot $\left(04^{\circ} 44^{\prime} 21^{\prime \prime} \mathrm{E}, 47^{\circ} 03^{\prime} 32^{\prime \prime} \mathrm{N}\right)$, niveau 96 moyen. Calcaire à gryphées. Sinémurien inférieur, chronozone à Semicostatum (sous-chronozone à Scipionianum ou à Sauzeanum) ou chronozone à Turneri (Corna \& Dommergues 1995).

DiAgNose. - Comme pour le genre.

\section{DESCRIPTION}

Le seul spécimen disponible est un moule interne correspondant à un phragmocône incomplet d'environ $26,5 \mathrm{~mm}$ de diamètre. Seuls quelques fragments du test sont préservés. La coquille, franchement comprimée $(\mathrm{E} / \mathrm{D} \leq 30 \%)$, est de type sub-oxycône avec un ombilic faiblement ouvert $(\mathrm{O} / \mathrm{D} \leq 20 \%)$ et un assez fort taux de recouvrement des tours successifs (c. 35\%). L'ombilic est peu profond. Le mur ombilical est discret mais assez bien individualisé. Il est presque perpendiculaire au plan de symétrie de la coquille et est séparé de la base des flancs par un épaulement qui correspond à une nette cambrure du test (Figs $3 ; 4 \mathrm{~A})$. Au-delà du rebord ombilical et jusque vers la moitié de la hauteur du tour, les flancs sont à peine bombés et presque parallèles. Ensuite, et en direction du ventre, ils deviennent progressivement de plus en plus convergents et conferent à l'aire ventrale une allure nettement ogivale. Au
A

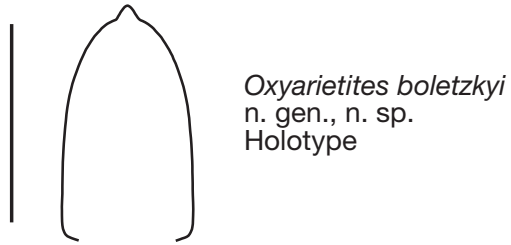

B

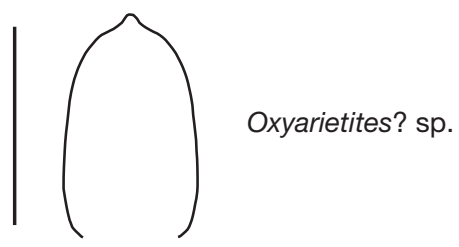

FIG. 4. - Profils schématiques des sections des tours d'Oxyarietites boletzkyi n. gen., n. sp. (A) et d'Oxyarietites? sp. (B). Ces profils ont été dessinés à partir de moulages en silicone. Les bandes périsiphonales assez visibles sous éclairage rasant chez $O$. boletzkyi n. gen., n. sp. (voir Figure 3F) sont à peine individualisées sur le profil de la section du tour (A).

plus grand des diamètres mesurables, la section des tours est franchement comprimée $(\mathrm{E} / \mathrm{H}=0,60)$. L'aire ventrale porte une carène bien développée et individualisée mais, au moins sur le moule interne, peu tranchante. La carène est bordée de bandes obliques, lisses, assez larges et discrètement concaves. La limite entre ces bandes et la partie supérieure des flancs correspond à une légère accentuation de la courbure du test qui est surtout visible en lumière rasante (Fig. 3B) mais qui apparaît à peine sur le profil de la section des tours (Fig. 4A).

Lornementation latérale est très discrète et le moule interne peut, à première vue, paraître presque lisse. Vers 15 à $18 \mathrm{~mm}$ de diamètre, il s'agit de bourrelets peu saillants, assez espacés, et surtout perceptibles en lumière rasante (Fig. 3A). Ces bourrelets, surtout visibles vers la partie inférieure des flancs, tendent à s'élargir et à s'effacer en direction du ventre. Ils disparaissent avant d'atteindre la partie supérieure des flancs qui est lisse. Au-delà de $20 \mathrm{~mm}$ de diamètre, ces bourrelets laissent la place à une costulation délicate un peu irrégulière et légèrement flexueuse. Les côtes, au tracé discrètement biconcave, tendent à se diviser sur la moitié inférieure des flancs (Fig. 3C, D). Elles disparaissent dans la partie supérieure des flancs et ne semblent pas atteindre la bordure des bandes périsiphonales. 
Plusieurs lignes de sutures successives sont visibles sur l'holotype qui est un moule interne phosphaté. L'une de ces lignes, particulièrement bien conservée, est illustrée sur la Figure 5A, B. La ligne est peu profondément indentée (divisions de premier ordre) et faiblement incisée (divisions de second ordre). Il n'y a pratiquement pas d'incisions de troisième ordre. Le tracé, d'aspect tendu, est globalement rétroverse. La forme des lobes (i.e. L et U2) est grossièrement triangulaire avec une structure confusément trifide. Les amplitudes des principaux lobes et selles (i.e. U2, 2SL, L, 1SL, E) augmentent discrètement mais régulièrement en allant de la partie médiane des flancs vers l'aire ventrale et sans qu'aucun de ces éléments ne s'écarte notablement de la tendance générale. Sur la partie interne des flancs, entre la suture ombilicale et le lobe U2, on observe un lobe suspensif remarquablement ample et particulièrement peu indenté. Il semble curieusement presque plat, d'allure presque arasée. Le lobe externe est relativement étroit et peu incisé avec une selle ventrale à peine développée.

\section{Oxyarietites? sp}

(Figs 3E, F ; 4B)

Agassiceras sp. - Corna \& Dommergues 1995: pl. 5, fig. 2.

MESURES. - Malgré une préservation imparfaite, une série incomplète de mesures a pu être effectuée (Tableau 2).

ÂGE ET DISTRIBUTION. — L'ammonite décrite ici est le seul spécimen qui puisse être rapproché avec réserves du genre Oxyarietites n. gen. Elle provient de la même coupe et du même banc (Mandelot, niveau 96 moyen) que l'holotype d'Oxyarietites boletzkyi n. gen., n. sp. Son âge correspond au Sinémurien inférieur, chronozone à Semicostatum (sous-chronozone à Scipionianum ou à Sauzeanum) ou chronozone à Turneri (Corna \& Dommergues 1995).

\section{DESCRIPTION}

Cet exemplaire est un moule interne d'aspect phosphaté, partiellement encroûté et corrodé, d'environ $28 \mathrm{~mm}$ de diamètre. Les lignes de sutures ne sont pas visibles et il est difficile de dire s'il s'agit d'un phragmocône plus ou moins complet ou d'un exemplaire pourvu de tout ou partie de la loge d'habitation. Il pourrait s'agir d'une forme micromorphe (taxon de petite taille ou dimorphe microconche) dont l'aspect légèrement extraombiliqué du dernier demi-tour de spire pourrait correspondre à la loge d'habitation d'un exemplaire adulte.

La coquille comprimée (E/D = 27\%) est d'un type intermédiaire entre les morphologies platycône et suboxycône. Il s'agit d'une forme plutôt involute avec un ombilic qui dépasse à peine $30 \%$ du diamètre de la coquille et un recouvrement assez important des tours successifs. L'ombilic est peu profond. Le mur ombilical est indistinct et le passage de l'aire ombilicale à la base des flancs s'effectue assez progressivement sans rebord ombilical bien différencié (Figs 3F ; 4B). Les flancs sont, sur les deux tiers inférieurs de la hauteur du tour, légèrement bombés et à peine convergents. Ils se cintrent ensuite assez rapidement en direction du ventre. La section du tour, subovale à subogivale, est franchement comprimée $(\mathrm{E} / \mathrm{H}=0,67)$. L'aire ventrale porte une carène peu élevée et peu tranchante. Elle est bordée de méplats obliques qui se différencient difficilement de la partie supérieure des flancs (Figs 3E; 4B). L'ornementation latérale, assez lâche et plutôt inconsistante, se réduit à quelques bourrelets peu individualisés. Ces bourrelets - une dizaine sur le dernier demi-tour - ne sont perceptibles qu'en lumière rasante et surtout vers le tiers médian des flancs (Fig. 3F).

\section{DISCUSSION}

LES AFFINITÉS D'OXYARIETITES N. GEN.

Oxyarietites boletzkyi n. gen., n. sp., unique espèce du genre Oxyarietites n. gen., est difficile à attribuer à une famille voire même à une super-famille. La coquille du nouveau taxon, presque oxycône et faiblement ornée, s'écarte, très nettement de celles, en général serpenticônes ou platycônes, des ammonites de l'Hettangien et du Sinémurien inférieur. Aucun argument stratophénétique (succession stratigraphiquement contrainte de formes intermédiaires) ne permet actuellement de guider le choix taxonomique. La présence d'une véritable carène bordée de deux méplats obliques plus ou moins bien individualisés est le seul caractère qui permette de suspecter une appartenance à la super-famille des Arietitoidea Hyatt, 1875 sensu Guex (1995). Ce type d'ornementation 

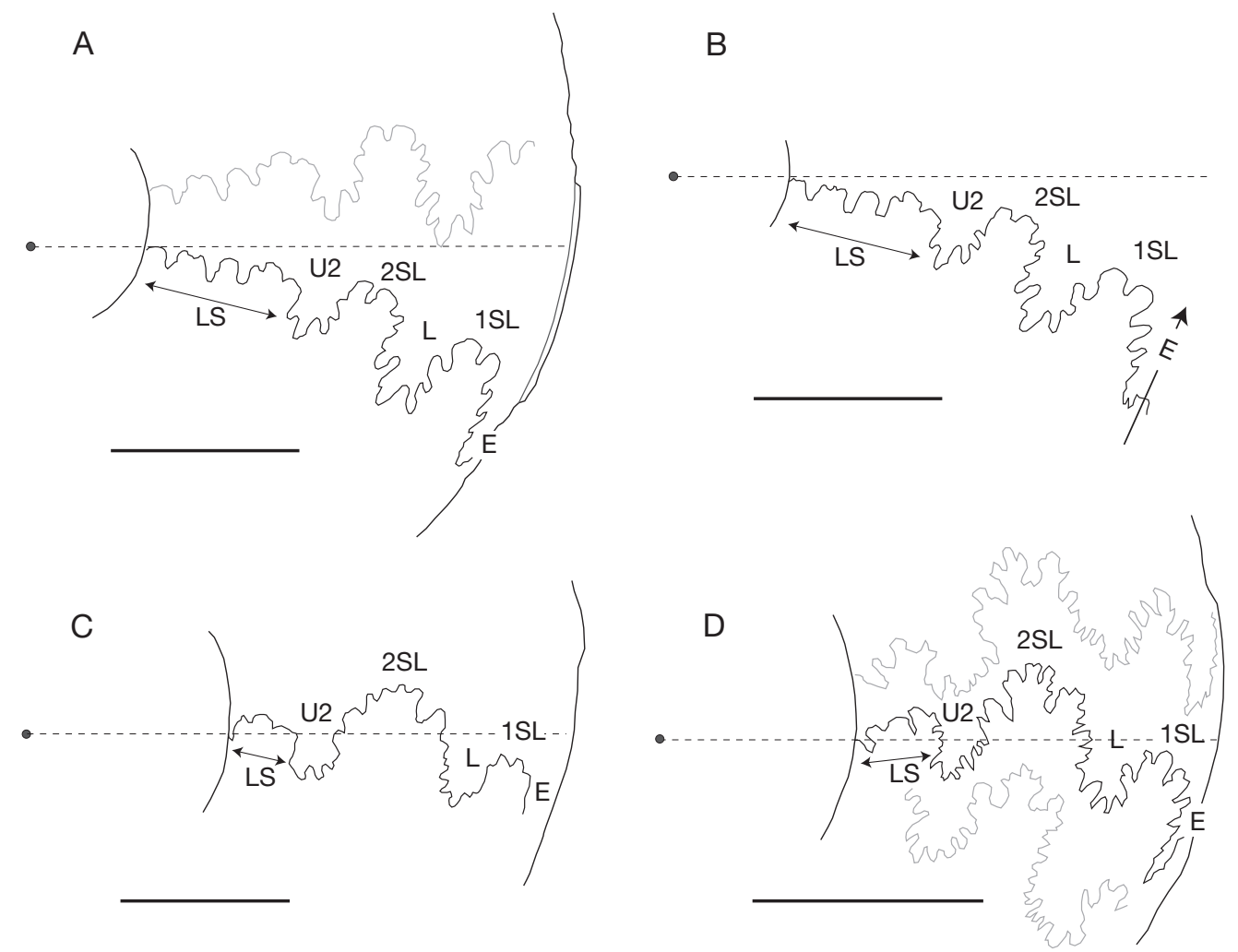

FIG. 5. - Comparaison des lignes de suture d'Oxyarietites boletzkyi n. gen., n. sp. et de deux espèces d'Agassiceras Hyatt, 1875: A, B, Oxyarietites boletzkyi n. gen., n. sp.: vue latérale (A) et vue déroulée (B) de l'holotype Mandelot (Côte-d'Or, France), niveau 96 moyen (UBGD 277446); C, Agassiceras cf. scipionianum (d'Orbigny, 1844), vue latérale, Saint-Jérôme (Ain, France), niveau 75 (STJ001); D, A. nodulatum (Buckman, 1921) vue latérale de la ligne de suture de holotype, modifiée d'après Buckman (1921: pl. 221), Newtown (Somerset, Grande Bretagne). Une ligne pointillée passant par la protoconche et le point d'intersection entre la ligne de suture et la suture ombilicale est indiquée pour permettre d'orienter la ligne de suture de chaque spécimen. Abréviations: LS, lobe suspensif (à l'exclusion de U2); U2, second lobe ombilical; 2SL, seconde selle latérale; L, lobe latéral; 1SL, première selle latérale; E, lobe externe. Échelles: A-C, $5 \mathrm{~mm}$; D, $50 \mathrm{~mm}$.

ventrale est en effet inconnu chez les autres superfamilles de l'Hettangien et du Sinémurien inférieur (e.g., Phylloceratoidea Zittel, 1884; Psiloceratoidea Hyatt, 1867 sensu Guex [1995]; Lytoceratoidea Neumayr, 1875). Au rang de la famille, on pourrait être tenté de rapprocher le nouveau taxon des Arietitidae Hyatt, 1875 et plus précisément de la sousfamille des Agassiceratinae Spath, 1924 qui caractérise la chronozone à Semicostatum (sous-chronozone à Scipionianum et à Sauzeanum). Chez le genre Agassiceras Hyatt, 1875, la croissance s'accompagne d'une augmentation de l'involution de la coquille, de sections des tours de plus en plus comprimées et ogivales ainsi que d'un affaiblissement progressif de la costulation. De plus, l'aire ventrale acquiert souvent une allure pincée renforcée par la présence d'une carène tranchante. Cette tendance évolutive peramorphique, qui s'exprime chez certains Agassiceratinae et en particulier au sein du genre Agassiceras, mène à des stades adultes assez involutes, mais son expression reste limitée. Les formes les plus dérivées atteignent au plus, et en fin de croissance, le type subplatycône involute. En outre, les tours internes de tels Agassiceras restent, en général, plutôt évolutes et portent habituellement une costulation simple et vigoureuse très différente de celle délicate, complexe 
et flexueuse d'Oxyarietites boletzkyi n. gen., n. sp. Rien ne démontre donc qu'Oxyarietites n. gen. soit phylétiquement proche des Agassiceratinae et plus généralement des Arietitidae. De plus, la ligne de suture d'Oxyarietites boletzkyi n. gen., n. sp. est clairement distincte de celles de tous les Arietitidae d'âges comparables ou proches (e.g., Coroniceras Hyatt, 1867 ; Paracoroniceras Spath, 1922; Pararnioceras Spath, 1922; Agassiceras; Euagassiceras Spath, 1924; Caenisites Buckman, 1925; et Asteroceras Hyatt, 1867). Pour illustrer ces différences, les lignes de sutures de deux spécimens d'Agassiceras, de tailles très différentes, sont données sur la Figure 5C, D. Contrairement à celle d'Oxyarietites n. gen. (Fig. 5A, B), les lignes d'Agassiceras sont profondément indentées et/ou incisées. Leur orientation est radiale entre la suture ombilicale et la seconde selle latérale (2SL) puis fortement rétroverse à l'approche de l'aire ventrale. Les selles (i.e. 1SL, 2SL) sont nettement plus amples que les lobes (i.e. L, U2) et la seconde selle latérale (2SL), d'allure proéminente, montre une forte projection adaperturale. Le lobe suspensif, d'aspect contracté se réduit au secteur proche de l'ombilic. La selle externe est bien développée au sein du lobe externe.

La seule ammonite, avec laquelle Oxyarietites boletzkyi n. gen., n. sp. puisse être comparée, provient également de la faune du niveau 96 moyen de la coupe de Mandelot. Cette ammonite, Oxyarietites? sp. (Fig. 3D, E), a été illustrée et décrite par Corna \& Dommergues (1995: pl. 5, fig. 2) sous la dénomination provisoire d'Agassiceras sp. Elle est rapprochée ici, avec réserve, du nouveau genre Oxyarietites n. gen. Cette forme présente un ombilic relativement petit, une section des tours subogivale, une carène bordée par de faibles méplats et une ornementation réduite à quelques bourrelets peu visibles. On pourrait interpréter, au moins à titre d'hypothèse de travail, ce petit spécimen (adulte?) comme un microconche associé au groupe d'O. boletzkyi n. gen., n. sp. Cette hypothèse ne facilite malheureusement pas l'interprétation des affinités taxonomiques du nouveau genre car, si Oxyarietites? sp. et $O$. boletzkyi n. gen., n. sp. sont deux formes morphologiquement assez proches, elles demeurent très éloignées de toutes les autres ammonites de l'Hettangien et du Sinémurien inférieur. Il faudrait étendre les comparaisons au Sinémurien supérieur pour trouver, au sein de la famille des Oxynoticeratidae Hyatt, 1875, des formes aux habitus proches de celui d'Oxyarietites boletzkyi n. gen., n. sp. Ces ressemblances ont un sens en termes morphologiques (convergences) mais elles ne sont pas pertinentes en termes de phylogénie car l'enracinement des Oxynoticeratidae au sein des Asteroceratinae (Caenisites, Asteroceras) est maintenant bien documenté (Donovan 1994). Par exclusion, Oxyarietites n. gen. ne peut donc pas être considéré comme un taxon étroitement apparenté aux Oxynoticeratidae. Il s'agit plutôt d'un représentant, au sein de la super-famille des Arietitoidea, d'une lignée indépendante ayant évoluée précocement, mais très discrètement, vers les morphologies oxycônes.

\section{L'ÉMERGENCE DES FORMES OXYCÔNES AU JURASSIQUE}

Il est actuellement largement admis que la géométrie des coquilles d'ammonites influe, directement et très significativement, sur les capacités de flottaison et de mobilité de ces céphalopodes. Les auteurs (e.g., Jacobs \& Chamberlain 1996; Westermann 1996; Klug \& Korn 2004; Klug et al. 2005; Oloriz et al. 2002) s'accordent en particulier pour admettre que les ammonites avec des coquilles involutes et comprimées de types suboxycône et oxycône possédaient les meilleures capacités de mobilité avec des déplacements précis, stables et sans doute relativement rapides. On peut donc supposer que l'acquisition de ce type de coquille apportait des avantages adaptatifs importants, en permettant, notamment, d'échapper plus facilement aux prédateurs. Il est donc surprenant de constater l'absence de coquilles oxycônes et la rareté de formes suboxycônes dans les séries fos-

FIG. 6. - Illustration schématique des principaux taxons exprimant des morphologies de types oxycônes ou suboxycônes au cours des trois premiers étages du Jurassique dans le nord-ouest de l'Europe et la Téthys méditerranéenne. Les Amaltheidae Hyatt, 1867 et Hildoceratidae Hyatt, 1867 oxycônes ou suboxycônes du Pliensbachien supérieur ne sont pas figurées. Les ammonites (dessinées sans échelle de taille) sont placées dans le cadre stratigraphique avec une précision de l'ordre de la sous-chronozone. Les taxons correspondant à des symboles pleins $(\bullet)$ ont des morphologies qui s'accordent bien au type oxycône, ceux marqués par des symboles creux $(\bigcirc)$ ont des habitus qui s'écartent par un ou quelques points de ce type morphologique. À titre d'exemple la coquille 


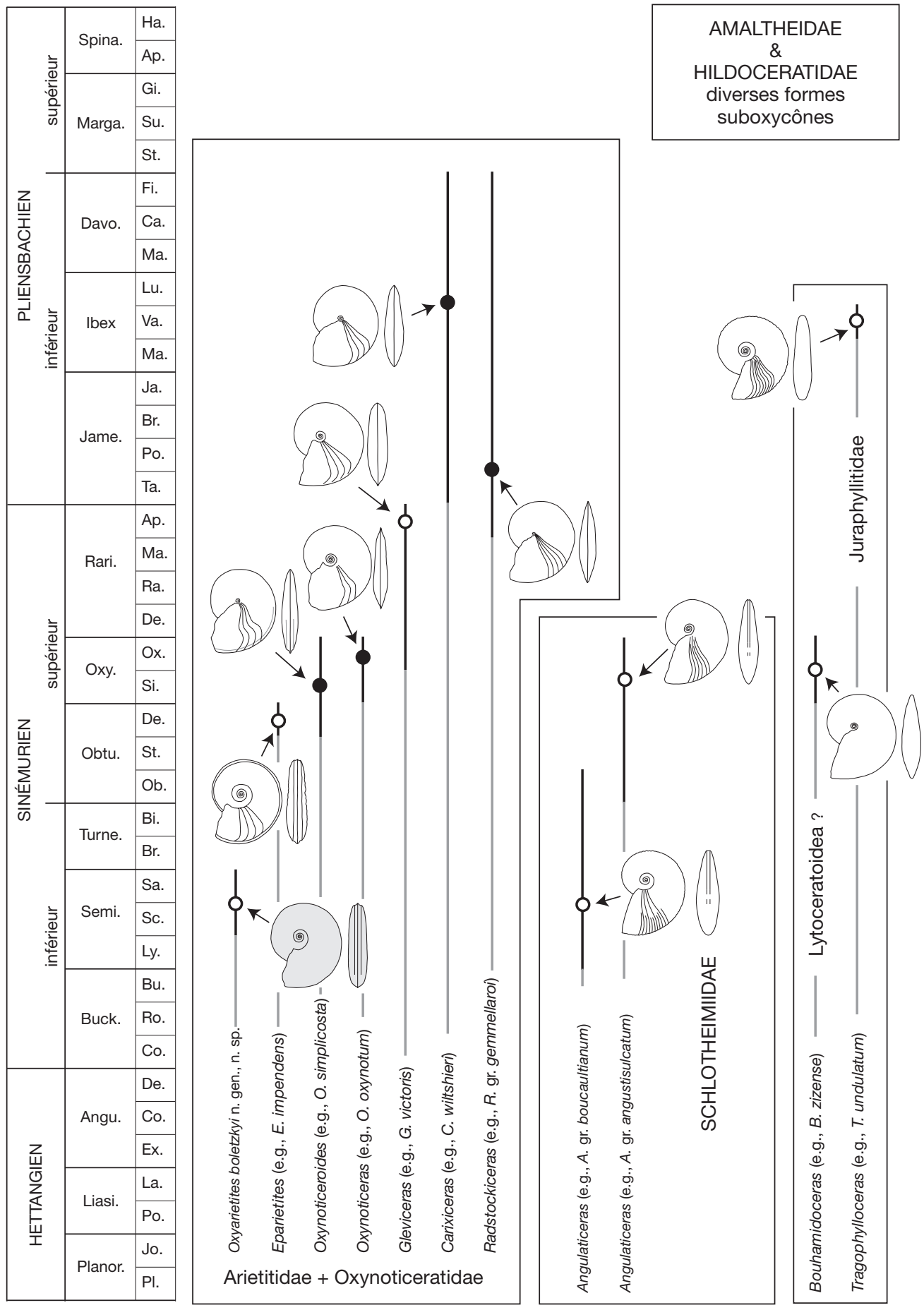

d'Angulaticeras angustisulcatum (Schlotheimiidae) possède une aire ventrale étroite d'allure presque pincée, mais elle est dépourvue d'une véritable carène. Échelle chronostratigraphique d'après Page (2003). 
siliferes de l'Hettangien et du Sinémurien inférieur (Dommergues et al. 1996) (Fig. 6). Il faut attendre environ huit millions d'années après la crise faunique Trias/Jurassique pour voir apparaître, au cours de la chronozone à Oxynotum (Sinémurien supérieur) et au sein de la famille des Oxynoticeratidae, les premières formes incontestablement oxycônes du Jurassique. La Figure 6 illustre et résume la distribution stratigraphique et le contexte taxonomique de l'histoire des formes suboxycônes et oxycônes au cours des trois premiers étages du Jurassique. L'essentiel de ces ammonites appartiennent aux Arietitidae et aux Oxynoticeratidae qui en descendent directement. En dehors de ces formes nettement carénées, on observe, au sein des Schlotheimiidae Spath, 1923, des Lytoceratoidea et des Juraphyllitidae Arkell, 1950, quelques espèces qui acquièrent des morphologies proches du type oxycône mais elles s'en différencient toujours par l'absence d'une véritable carène.

Les toutes premières formes suboxycônes du Jurassique apparaissent au cours de la chronozone à Semicostatum. Il s'agit d'Angulaticeras Quenstedt, 1883 du groupe d'A. boucaultianum (Orbigny, 1844) (Schlotheimiidae) et d'Oxyarietites boletzkyi n. gen., n. sp., sans doute assez proche des Arietitidae. Ce nouveau taxon est donc, au moins en termes morphologiques, un véritable précurseur pour les formes oxycônes carénées qui deviendront abondantes au cours du Sinémurien supérieur. L'extrême rareté d'O. boletzkyi n. gen., n. sp. est, à bien des égards, remarquable. Sa découverte comble en tout cas une lacune importante dans la connaissance des faunes d'ammonites du nord-ouest de l'Europe pourtant très étudiées et relativement bien connues. Il est surprenant qu'un taxon caractérisé par une morphologie aussi typée qu'O. boletzkyi n. gen., n. sp. ait pu, jusqu'à présent, demeurer inconnu et que son origine phylétique reste une énigme. Il est aussi intéressant de souligner que l'acquisition d'une coquille presque oxycône, sans doute associée à de bonnes performances hydrodynamiques, ne soit pas corrélée à une certaine abondance au sein des peuplements. Dans le cas d'O. boletzkyi n. gen., n. sp., il est impossible de prouver l'existence de relations claires entre innovation morphologique, adaptation et succès évolutif.

\section{Remerciements}

Ce travail est une contribution de l'équipe BioME «Biodiversité, Macroécologie, Évolution » de l'UMR 6282, «Biogéosciences» (CNRS/uB). L'un des auteur (SG) tient à remercier les organisateurs $d u$ « $8^{\text {th }}$ International Symposium, Cephalopods - Present and Past» (31 août-3 septembre 2010, Dijon, France), et surtout les guides Pascal Neige, Jean-Louis Dommergues et Emmanuel Fara du «Fieldtrip 1 : A day in Burgundy» (4 septembre) au cours duquel a été récolté le spécimen holotype du nouveau taxon. Les auteurs remercient également A. F. Bannikov, F. Santini et A. Ohler, qui ont lu une première version du manuscrit.

\section{RÉFÉRENCES}

BucKman S. S. 1919-1930. - Type Ammonites. Wheldon \& Wesley, London. Vols 3-7: pls 131-790.

COLLENOT J.-J. 1873. - Description géologique de l'Auxois. Semur-en Auxois, 660 p.

Collenot J.-J. 1879. — Description sommaire des terrains sédimentaires de l'Auxois. Bulletin de la Société géologique de France (Série 3) 7: 781-804.

Corna M. \& Mouterde R. 1988. — Le Sinémurien de Semur (France) : essai de biozonation pour le Sinémurien inférieur et le Lotharingien inférieur du stratotype et du Jura méridional, in RocHA R. B. \& SOARES A. F. (éds), 2nd international Symposium on Jurassic Stratigraphy, Lisboa: 155-178.

Corna M. \& Dommergues J.-L. 1995. - Les ammonites du Sinémurien de Mandelot (Côte-d'Or, France). Approches biostratigraphique, morphologique et ontogénétique. Geobios 28: 17-47.

Corna M., Dommergues J.-L., Meister C., Mouterde R. \& Bloos G. 1997. - Sinémurien, in Cariou E. \& HANTZPERGUe P. (éds), Biostratigraphie du Jurassique ouest-européen et méditerranéen : zonations parallèles et distribution des invertébrés et microfossiles. Bulletin des Centres de Recherche Elf Exploration et Production, Mémoire 17: 15-23.

Dommergues J.-L. 1993. — Les ammonites du Sinémurien supérieur de Bourgogne (France): biostratigraphie et remarques paléontologiques. Revue de Paléobiologie 12: 67-173.

Dommergues J.-L., Laurin B. \& Meister C. 1996. Evolution of ammonoid morphospace during the Early Jurassic radiation. Paleobiology 22: 219-240.

Dommergues J.-L., Laurin B. \& MeISTer C. 2001. The recovery and radiation of Early Jurassic ammonoids: morphologic versus palaeobiogeographical patterns. Palaeogeography, Palaeoclimatology, Palaeoecology 165: 195-213. 
Dommergues J.-L., Montuire S. \& Neige P. 2002. Size patterns through time: the case of the Early Jurassic ammonite radiation. Paleobiology 28: 423-434.

Donovan D. T. 1994. - Evolution in some Early Jurassic ammonites: Asteroceratinae, Oxynoticeratidae and related forms, in Pallini G. (éd.), Proccedings of the 3rd Pergola International Symposium "Fossili, Evolution, Ambiente", October 1990. Palaeopelagos special publication 1: 383-396.

FISCHER J.-C. (éd.) 1994. — Révision critique de la paléontologie française d'Alcide d'Orbigny. Vol. 1. Céphalopodes jurassiques. Masson \& Muséum national d'Histoire naturelle, Paris, $340 \mathrm{p}$.

Guérin-Franiatte S. 1966. - Ammonites du Lias inférieur de France. Psiloceratidae: Arietitidae. Centre national de la Recherche scientifique (CNRS), Paris, $455 \mathrm{p}$.

Guérin-Franiatte S. 1990. - Ammonites du Lias inférieur de France (II) : Psiloceratidae, Schlotheimiidae et premiers Arietitidae. Mémoire du Centre d'étude et de recherches géologiques et hydrologiques 29: 1-207.

GuEX J. 1982. - Relation entre le genre Psiloceras et les Phylloceratida au voisinage de la limite Trias-Jurassique. Bulletin de Géologie, Lausanne 260: 47-51.

GueX J. 1987. - Sur la phylogenèse des ammonites du Lias inférieur. Bulletin de Géologie, Lausanne 292: 455-469.

GueX J. 1995. - Ammonites hettangiennes de la Gabbs Valley Range (Nevada, USA). Mémoires de Géologie, Lausanne 27, 131 p.

GueX J., TAYlor D., Rakus M. \& Bucher H. 1998. Deux nouveaux genres et quatre nouvelles espèces d'ammonites (Cephalopoda) du Lias inférieur. Bulletin de Géologie, Lausanne 339: 73-85.

Hillebrandt A. VON \& Krystin L. 2009. - On the oldest Jurassic ammonites of Europe (northern Calcareous Alps, Austria) and their global significance. Neues Jahrbuch für Geologie und Paläontologie, Abhandlungen 253: 163-195.

HofFMANN R. 2010. - New insights on the phylogeny of the Lytoceratoidea (Ammonitina) from the septal lobe and its functional interpretation. Revue de Paléobiologie 29: 1-156.

Jacobs D. K. \& Chamberlain J. A. 1996. - Buoyancy and hydrodynamics in ammonoids, in LANDMAN N., TANABE K. \& DAVIS R. A. (éds), Ammonoid Paleobiology. Topics in Geobiology. Vol. 13. Plenum, New York: 43-63.

KLUG C. \& Korn D. 2004. - The origin of ammonoid locomotion. Acta Palaeontologica Polonica 49: 235-242.

Klug C., Schatz W., Korn D. \& Reisdorf A. 2005. Morphological fluctuations of ammonoid assemblages from the Muschelkalk (Middle Triassic) of the Ger- manic basin - indicators of their ecology, extinctions, and immigrations. Palaeogeography, Palaeoclimatology, Palaeoecology 221: 7-34.

Mouterde R. 1953. - Études sur le Lias et le Bajocien des bordures nord et nord-est du Massif central français. Bulletin du Service de la Carte géologique de France 50 (1952) 236, $521 \mathrm{p}$.

Mouterde R. \& Tintant H. 1961. - Le Sinémurien de Semur, in Colloque sur le Lias français, Chambery 1960. Mémoires du Bureau de Recherches géologiques et minières (BRGM) 4: 287-295.

Mouterde R. \& Tintant H. 1964. - Variations du Sinémurien dans la région du stratotype (précisions sur la notion d'étage), in Maubeuge P. L. (éd.), Colloque du Jurassique (Luxembourg 1962). Publications de l'Institut Grand-Ducal, Luxembourg: 119-126.

Mouterde R. \& Tintant H. 1980. - Sinémurien, in Cavelier C. \& Roger J. (éds), Les étages français et leur stratotypes. Mémoires du Bureau de Recherches géologiques et minières (BRGM) 109: 50-58.

Oloriz F., PalmQVist P. \& Pérez-Claros J. A. 2002. Morphostructural constraints and phylogenetic overprint on sutural frilling in Late Jurassic ammonites. Lethaia 35: 158-168.

Orbigny A. D' 1844. - Paléontologie française: terrains jurassiques. I. Céphalopodes. Livres 17-27. Masson, Paris: 193-312.

OrbignY A. D' 1849-1852. - Cours élémentaire de paléontologie et de géologie stratigraphique. Masson, Paris. Vol. I (1849): 1-299; Vol. II (1851): 1-382; Vol. III (1852): 383-847.

PAGE K. 2003. - The Lower Jurassic of Europe: its subdivision and correlation. Geological Survey of Denmark and Greenland Bulletin 1: 23-59.

RaKUS M. 1993. — Late Triassic and Early Jurassic phylloceratids from the Salzkammergut (northern Calcareous Alps). Jahrbuch der Geologischen Bundesanstalt 136: 933-963.

Simon M. S., Korn D. \& Koenemann S. 2010. — Disparity fluctuations in Jurassic ammonoids by means of conch geometry. Palaeogeography, Palaeoclimatology, Palaeoecology 292: 520-531.

TOZER. E.T. 1981. - Triasic ammonoidea: classification evolution and relationship with Permian and Jurassic forms, in House M. R. \& SENIOR J. R. (éds), The Ammonoidea. The Evolution, Classification, Mode of Life and Geological Usefulness of a Major Fossil Group. Academic Press, London: 101-155.

WestermanN G. E. G. 1996. - Ammonoid life and habitat, in Landman N., TANABE K. \& DaVIs R. A. (éds), Ammonoid Paleobiology. Topics in Geobiology 13: 607-707. 